\title{
Truth and consequences - The issue with standards and guidelines
}

\author{
Martin A. Koyle, MD, MSc \\ Division of Pediatric Urology, Hospital for Sick Children, University of Toronto \\ Toronto, ON, Canada
}

Cite as: Can Urol Assoc J 2018 Feb.12; Epub ahead of print. http://dx.doi.org/10.5489/cuaj.5237

\section{Published online February 12, 2018}

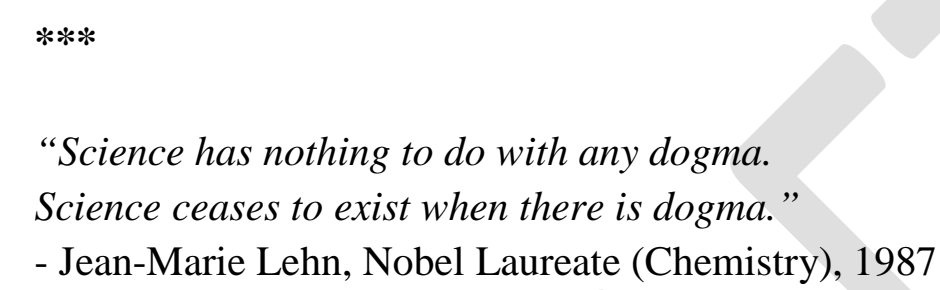

As the world of medicine turns, emphasis has been placed on performance and costeffective, evidence-based care. Value, quality divided by $\operatorname{costs}(\mathrm{V}=\mathrm{Q} / \$)$, has therefore become a major driver in modern healthcare. (Donald) Berwick and the Institute for Healthcare Improvement (IHI) have promoted this concept in their triple aim of improved experience of care, with improved health of populations, while reducing costs of healthcare.

In our specialty, many of our practices are still largely based on our past training, and the influence upon us of our mentors, that is, "experience-based medicine.” Whereas my training, completed over three decades ago, was paternalistic — where the doctor was (almost) always right (and dogmatic about it) - we are now in an era of shared decisionmaking, where even in non-surgical situations, by offering options (i.e., surveillance rather than surgical intervention of a disease process such as prostate cancer or vesicoureteral reflux), we essentially are using the informed consent process. Savvy patients come to our clinics armed with the newest advances posted on the Web or described in the media. Today's medical school graduates who enter urology have been tutored in resource stewardship, while residents are partially evaluated in the leadership category by understanding and addressing these issues. It thus behoves specialty societies to encourage standards to be advocated for and established.

Clinical practice guidelines (CPGs), in essence, should be based on systematic reviews (SR) of current best evidence regarding a given topic, evidence that has been addressed by an unbiased, multidisciplinary group of experts, so that it can used to assist practitioners and their patients in optimizing patient care. Such CPGs must be well 
thought out and continuously updated in order to assure their relevance and sustainability based on evidence-based medicine (EBM). David Sackett's description of EBM is most apt: "The conscientious, explicit, and judicious use of current best evidence in making decisions about the care of individual patients." Problematic is the reality that not all EBMs have been based on high-quality evidence, which potentially can lead to less than ideal results, and thus has led to scepticism regarding their implementation. ${ }^{1}$

Our practices are busy and this makes it complicated to be knowledgeable about the most up-to-date/state-of-the-art issues, as disruptive technology and advances in all aspects of healthcare grow exponentially. CPGs should take voluminous and/or complex data that isn't at our fingertips and make such data more manageable for that individual patient, who is cared for by that individual physician, at that given time. Hence, in no way should CPGs be construed as cookbook medicine or the only option for that specific patient or encounter. Sadly, defensive medicine and "over-testing" are realities of the practice of medicine. This is costly to the system as a whole and impacts the utility of CPGs and other progressive initiatives, such as "Choosing Wisely."

Medicine continues to remain an art, where one's experience and know-how are still essential. It seems far more likely that older, "independent” physicians (like me), with my training and experience (and built-in biases) would be less likely to embrace CPGs than recent, "team-based" graduates who have been brought up in a culture where guidelines are considered the norm. In fact, especially to those like me for whom CPGs have not been our vocabulary, too many CPGs are viewed as boring and lengthy, with unnecessary tables and graphs, and conflicting recommendations that make their acceptance a challenge. This is potentially exacerbated by knowledge of who sponsored the CPG (medical society or organization, hospital, industry), the incentives that might promote the CPG, and the region from which it is developed, as well as the composition of the guidelines committee itself. Might the electronic medical record (EMR) ultimately make implementation of CPGs simpler by having them more accessible and also by measuring adherence to them, i.e., by making their use more of an active rather than a passive component? I would wager that this will be the case.

GRADE (Grades of Recommendation, Assessment, Development, and Evaluation) has been developed as tool for constructing CPGs that enhance physician evaluation of the quality of medical evidence so that recommendations can be applied most appropriately in patient care. ${ }^{2}$ It replaces letter recommendations regarding quality with "strong” or "weak/conditional"/“for" or "against", and avoids "expert opinion" in the hierarchy of the evidence pyramid, while recognizing that even the highest evidence standard, SR, can be flawed. Recommendations using GRADE ideally should be actionable and avoid restating obvious facts and vagaries.

The AGREE II tool (Appraisal of Guidelines for Research and Evaluation) has gained acceptance as a standardized technique for the grading of CPGs. ${ }^{3,4}$ It consists of 
six quality domains containing 23 items used to quantify each CPG. It requires several appraisers who independently score each domain of each guideline. Importantly each appraiser also assesses the overall quality of the CPGs and makes a recommendation as to whether that guideline should be used.

CPGs will increasingly be available to us. As such, practitioners must understand how they are developed and what their qualities and relevance are, in order to use them appropriately in selected circumstances. EMRs likely will improve their availability, as well as be an adjunct in improving compliance with their use. As such, CPGs should be used judiciously, remembering that medical judgment and experience remain essential components of our fostering shared decision-making and optimal care.

\section{References}

1. Sackett DL, Rosenberg WM, Gray JA, et al. Evidence-based medicine: What it is and what it isn't. BMJ 1996;312:71-2. https://doi.org/10.1136/bmj.312.7023.71

2. Guyatt GH, Oxman AD, Vist G, et al. GRADE: An emerging consensus on rating quality of evidence and strength of recommendations. BMJ 2008;336:9246. https://doi.org/10.1136/bmj.39489.470347.AD

3. Brouwers MC, Kho ME, Browman GP, et al. AGREE II: Advancing guideline development, reporting, and evaluation in healthcare. CMAJ 2010;182:83942. https://doi.org/10.1503/cmaj.090449

4. Chua M, Ming J, Chang S-J, et al. A critical review of recent clinical practice guidelines for pediatric urinary tract infection. Can Urol Assoc J 2017 Dec. 22; Epub ahead of print. http://dx.doi.org/10.5489/cuaj.4796 